\title{
INTEGRITY BASIS FOR A SECOND-ORDER AND A FOURTH-ORDER TENSOR
}

\author{
JOSEF BETTEN \\ Rheinisch-Westfälische Technische Hochschule Aachen \\ Templergraben 55 \\ D-5100 Aachen \\ Federal Republic of Germany \\ (Received July 8, 1981)
}

ABSTRACT. In this paper a scal.ar-valued isotropic tensor function is considered, the variables of which are constitutive tensors of orders two and four, for instance, characterizing the anisotropic properties of a material. Therefore, the system of of irreducible invariants of a fourth-order tensor is constructed. Furthermore, the joint or simultaneous invariants of a second-order and a fourth-order tensor are found. In a similar way one can construct an integrity basis for a tensor of order greater than four, as shown in the paper, for instance, for a tensor of order six.

KEY WORDS AND PHRASES. Theory of algebraic invariants, integrity basis under a subgroup, isotropic tensor functions, representation - theory, irreducible basic and principal invariants of a fourth-order tensor, constitutive tensors, char iteristic polynomial, alternation process, integrity basis for a tensor of orde $i$ greater than four, bilinear operator, construction of simultaneous or joint invariants, Hamilton-Cayley's theorem, isotropic constitutive tensors.

1980 MATHEMATICS SUBJECT CLASSIFICATION CODE. 53A45, $73 B$.

\section{INTRODUCTION.}

In many branches of mathematics and physics, for instance, in continuum mechanics, the central problem is: For a given set of tensors which are not necessarily of the same order, and a given group of transformations, find an integrity basis, the elements of which are algebraic invariants.

Many mathematicians have studied the theory of algebraic invariants in detail. The results are published, for instance, by GRACE and YOUNG [1], GUREVICH [2], 
WEITZENBÖCK [3], WEYL [4]. Very extensive accounts of algebraic invariant theory from the point of view of its application to modern continuum mechanics are presented, for example, by SPENCER [5], TRUESDELL and NOLL [6].

It is convenient to employ these results of the theory of invariants in the mechanics of isotropic and anisotropic materials [7]. In the theory of algebraic invariants the central problem is: For a given set of tensors which are not necessarily of the same order, and a given group of transformations, find an integrity basis, the elements of which are algebraic invariants. An integrity basis is a set of polynomials, each invariant under the group of transformations, such that any polynomial function invariant under the group is expressible as a polynomial in elements of the integrity basis.

In continuum mechanics, a constitutive expression may be a polynomial function which is appropriate for the description of the response of an anisotropic material. The representation of such an expression is based upon an integrity basis.

In this paper the system of irreducible (basic and principal) invariants of a fourth-order tensor is found. Furthermore, the joint or simultaneous invariants of a second-order and a fourth-order tensor are constructed.

The mentioned systems of invariants cannot be found in the cited literature. Therefore, the results of this paper are important, for instance, for a reader who is working in the field of theoretical continuum mechanics and who is familiar with tensor calculus.

2. INTEGRITY BASIS UNDER A SUBGROUP.

Let $F=F(\underset{\sim}{)}$ be a scalar-valued function of a second-order tensor, for instance, of the stress tensor $g$. This function is said to be isotropic if the condition

$$
F\left(a_{i p} a_{j q} \sigma_{p q}\right) \equiv F\left(\sigma_{i j}\right)
$$

is fulfilled under any orthogonal transformation $\left(a_{i k} a_{j k}=\sigma_{i j}\right)$, where the summation convention is utilized, and $\underset{\sim}{\delta}$ represents KRONECKER's tensor.

For example, the function $F$ in (2.1) may be the plastic potential. Then, from 
the theory of isotropic tensor functions $[5,7,8]$, it is evident that in an isotropic medium the plastic potential $\mathrm{F}$ can be expressed as a single-valued function of the irreducible basic invariants

$$
s_{\nu} \equiv \operatorname{tr} \sigma^{\nu} ; \nu=1,2,3
$$

or, alternatively, of the irreducible principal invariants

$$
J_{1} \equiv \sigma_{i i}, J_{2} \equiv-\sigma_{i[i]} \sigma_{j[j]}, J_{3} \equiv \sigma_{i[i]} \sigma_{j[j]} \sigma_{k[k]} \quad(2.3 a, b, c)
$$

of the stress tensor $\underset{\sim}{\sigma}$ that is

$$
\mathrm{F}=\mathrm{F}\left[S_{\nu}(\underset{\sim}{\sigma})\right] \quad \text { or } \quad \mathrm{F}=\mathrm{F}\left[\mathrm{J}_{\nu}(\underset{\sim}{\sigma})\right], \quad \nu=1,2,3 \quad(2.4 \mathrm{a}, \mathrm{b})
$$

respectively. In (2.3) the operation of alternation is used. This process is indicated by placing square brackets around those indices to which it applies, that is, the $\nu$ bracketed indices $i \ldots k$ are permuted in all possible ways, while indices which are excluded from the alternation are not bracketed. They keep their positions. Thus, we obtain $v$ ! terms. The terms corresponding to even permutations are given a plus sign, those which correspond to odd permutations a minus sign, and they are then added and divided by $\nu !$. Comparing (2.2) and (2.3), we find the relations :

$$
\mathrm{J}_{1}=\mathrm{s}_{1}, \mathrm{~J}_{2}=\left(\mathrm{s}_{2}-\mathrm{s}_{1}^{2}\right) / 2, \mathrm{~J}_{3}=\left(2 \mathrm{~s}_{3}-3 \mathrm{~s}_{2} \mathrm{~s}_{1}+\mathrm{s}_{1}^{3}\right) / 6 \quad(2.5 \mathrm{a}, \mathrm{b}, \mathrm{c})
$$

The irreducible (basic or principal) invariants are the elements of the integrity basis for the orthogonal group: An integrity basis is a set of polynomials, each invariant under the group of transformations, such that any polynomial function invariant under the group is expressible as a polynomial in the elements of the integrity basis $[4,5,9]$. The invariants $(2.2)$ or, alternatively, $(2.3 a, b, c)$ form the integrity basis for the stress tensor $\underset{\sim}{\sigma}$ under the proper orthogonal group, i.e. $\left|a_{i j}\right|=+1$, and this integrity basis also forms a functional basis.

The representations $(2.4 \mathrm{a}, \mathrm{b})$ imply isotropy.

In the anisotropic case the restriction (2.1) of $F$ is less severe. Then, the function $\mathrm{F}$ is merely required to be invariant under the group of transformations $\left(s_{i k} s_{j k}=\delta_{i j}\right)$ associated with the symmetry properties of the material [8], where $\mathbf{s}$ is a subgroup of the orthogonal group $\underset{\sim}{a}$. In other words, the symmetry properties 
of the material impose restrictions upon the manner in which the function $F$ depends on the stress components [10].

For a particular crystal class [11] the potential $\mathrm{F}$ may be represented as a polynomial in the stresses which is invariant under the subgroup $\$$ of transformations associated with the symmetry properties of the crystal class considered. The function $\mathrm{F}$ is then expressible as a polynomial in these invariants, which form a functional basis.

It is shown by PIPKIN and RIVLIN [12] and PIPKIN and WINEMANN [13] that an integrity basis will also form a functional basis if the group of transformations is finite; then all invariants can be expressed as functions of the invariants of an integrity basis.

3. INTEGRITY BASIS FOR A FOURTH-ORDER TENSOR.

Instead of the representation by an integrity basis under a subgroup anisotropic behaviour may be characterized by a function

$$
F=F\left(\sigma_{i j}, A_{i j}, A_{i j k 1}, A_{i j k 1 m}\right)
$$

in which $A_{i j}, A_{i j k l}$ etc. are the components of constitutive tensors characterizing the anisotropic properties of the material. Then, by analogy to (2.1), we have the invariance condition

$$
F\left(a_{i p} a_{j q}^{\sigma}{ }_{p q} ; \ldots, a_{i p} a_{j q} a_{k r} a_{1 s} A_{p q r s}, \ldots\right) \equiv F\left(\sigma_{i j} ; \ldots, A_{i j k 1}, \ldots\right)
$$

and the central problem is: to construct an irreducible integrity basis for the tensors $\sigma_{i j}, A_{i j}, A_{i j k l}, \ldots$ Together with the invariants of the single argument tensors $\sigma_{i j}, A_{i j}, A_{i j k 1}$, etc., like (2.2) or (2.3), we have to consider the system of simultaneous or joint invariants found in section 4.

Let us first construct the irreducible principal invariants of a fourth-order tensor $A_{i j k 1}$, which may be a linear operator, i.e.,

$$
Y_{i j}=A_{i j k 1} X_{k 1} \text { or } Y_{\alpha}=A_{\alpha \beta} X_{\beta} \text {, }
$$

where $i, j, k, 1=1,2,3$ or $\alpha, \beta=1,2, \ldots, 9$, respectively.

In (3.3b) the operator $\underset{\sim}{A}$ defines a linear transformation on a 9-dimensional vector space $v_{9}$, which is a correspondence that assigns to every vector $\underset{\sim}{X}$ in $v_{9} a$ 
vector $\underset{\sim}{\mathrm{AX}}$ in $\mathrm{V}_{9}$, in such a way that

$$
\underset{\sim}{A}\left(a_{1} \underset{\sim}{X_{1}}+a_{2} \underset{X_{2}}{\sim}\right)=a_{1} \underset{\sim}{A} \mathbb{X}_{1}+a_{2} \underset{\sim}{A X_{2}}
$$

identically in the vectors $\underset{\sim}{\mathrm{x}}$ and $\underset{\sim}{\mathrm{x}}$ and the scalars $a_{1}$ and $a_{2}$.

Let $\mathrm{x}_{\alpha} ; \alpha=1,2, \ldots, 9$; be the components of an arbitary vector of unit magnitude which we call direction vector or simply a direction. We then ask: For what directions $X$ does the linear transformation $\underset{\sim}{A}$ yield a vector $\underset{\sim}{\mathrm{a}}$ according to (3.3) which is in the same direction as $\mathrm{X}$ ? That is,

$$
\left(A_{i j k 1}-\lambda A_{i j k 1}^{(0)}\right) x_{k 1}=0_{i j} \quad \text { or } \quad\left(A_{\alpha \beta}-\lambda A_{\alpha \beta}^{(0)}\right) x_{\beta}=0_{\alpha} \quad(3.4 a, b)
$$

where $\lambda$ is a real scalar to be determined, $A_{i j k 1}^{(0)} \equiv \delta_{i k j 1} \delta_{j 1}$ or $A_{\alpha \beta}^{(0)} \equiv \delta_{\alpha \beta}$

are the components of the unit tensor $\underset{\sim}{\mathrm{A}}(0)$, whereas $0_{i j}$ or $0_{\alpha}$ are the components of the zero tensor 0 .

For a nontrivial solution of $(3.4 a, b)$ we must have

$$
\operatorname{det}\left(A_{i j k 1}-\lambda A_{i j k 1}^{(0)}\right)=0 \text { or } \operatorname{det}\left(A_{\alpha \beta}-\lambda A_{\alpha \beta}^{(0)}\right)=0
$$

in order to determine the principal or proper values $\lambda_{n}$ of the linear transformation $\underset{\sim}{\mathrm{A}}$.

In order to construct the principal invariants of a fourth-order tensor $\underset{\sim}{A}$, we note that $t^{-}$- Meterminant (3.5) is an invariant, and we therefore consider the characteristte ${ }^{\prime} \cdot$ 'ynomial

$$
r_{n}(\lambda) \equiv \operatorname{det}\left(A_{i j k 1}-\lambda A_{i j k 1}^{(0)}\right)=\sum_{\nu=0}^{n} J_{\nu}\left(\underset{\sim}{(A)} \lambda^{n-v}\right.
$$

in which, as we see from $(3.5 a, b)$, the first ind $x$ pair $(i j) \equiv \alpha$ characterizes the rows and the second one $(k 1) \equiv \beta$ the columns of $a n \times n$ matrix $\underset{\sim}{A}$, in general $n=9$.

The principal invariants $\mathrm{J}_{\nu}$ in (3.6) can be determined, analogous to (2.3), by the operation of alteration:

$$
(-1)^{n-v} J_{v} \equiv A_{\alpha_{1}\left[\alpha_{1}\right]} A_{\alpha_{2}}\left[\alpha_{2}\right] \cdots A_{\alpha_{v}}\left[\alpha_{v}\right]
$$

where $(-1)^{n} J_{0} \equiv 1$. The right hand side in (3.7) is equal to the sum of all $\left(\begin{array}{l}n \\ v\end{array}\right)=\frac{n !}{\nu !(n-v) !}$ principal minors of order $\nu \leq n$, where $\nu=1$ and $\nu=n$ lead to trA and det $\underset{\sim}{A}$, respectively. 
Assuming the usual symmetry conditions

$$
A_{i j k 1}=A_{j i k 1}=A_{i j l k}=A_{k l i j}
$$

or alternatively expressed by

$$
A_{\alpha \beta}=A_{B \alpha}, \quad \alpha, \beta=1,2, \ldots, 6
$$

the zero power tensor of fourth-order in (3.6) is given by:

$$
A_{i j k 1}^{(0)} \equiv A_{i j p q} A_{p q k 1}^{(-1)}=\frac{1}{2}\left(\delta_{i k} \delta_{j 1}+\delta_{i 1} \delta_{j k}\right)=A_{i j p q}^{(-1)} A_{p q k 1} \text {, }
$$

as we can see from (3.3) for $\underset{\sim}{\mathrm{Y}}=\underset{\sim}{\mathrm{X}}$

Expanding (3.7) with $\mathrm{n}=6$, we find the irreducible principal invariants of a fourth-order tensor by the alternation process already applied in (2.3):

$$
\begin{aligned}
& \mathrm{J}_{1} \equiv-A_{i j i j} \equiv-\operatorname{tr} \underset{\sim}{A} \\
& J_{2} \equiv \frac{1}{2 !}\left(A_{i j i j} A_{k 1 k 1}-A_{i j k 1} A_{k 1 i j}\right) \\
& J_{3} \equiv-\frac{1}{3 !}\left(A_{i j i j} A_{k 1 k 1} A_{m n m}+2 A_{i j k 1} A_{k 1 m n} A_{m n i j}-3 A_{i j k 1} A_{k 1 i j} A_{m n m}\right) \\
& \mathrm{J}_{4} \equiv \frac{1}{4 !}\left(A_{i j i j} A_{k l k l} A_{m n m n} A_{o p o p}+\right. \\
& 8 A_{i j k l} A_{k l m n} A_{m n i j} A_{o p o p}-6 A_{i j k l} A_{k l i j} A_{m n m n} A_{o p o p}- \\
& \left.6 A_{i j k l} A_{k l m n} A_{m n o p} A_{o p i j}+3 A_{i j k 1} A_{k l i j} A_{m n o p} A_{o p m n}\right) \\
& J_{5} \equiv-\frac{1}{5 !}\left(A_{i j i j} A_{k l k 1} \ldots A_{q r q r}+\ldots\right) \\
& J_{6} \equiv \frac{1}{6 !}\left(A_{i j i j} A_{k 1 k 1} \ldots A_{s t s t}+\ldots\right) \equiv \operatorname{det} \underset{\sim}{A} \text {. }
\end{aligned}
$$

We see that the principal invariants (3.10) of a fourth-order tensor can be determined uniquely by polynomial relations from the irreducible basic invariants

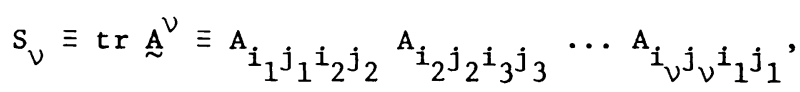

that is, by analogy to $(2.5)$,

$$
\mathrm{J}_{v}=\mathrm{P}_{\mathrm{v}}\left(\mathrm{S}_{1}, \mathrm{~S}_{2}, \ldots, \mathrm{S}_{\mathrm{v}}\right)
$$

Both the set of the six quantities (3.10) or, alternatively, the six quantities (3.11) form an irreducible integrity basis for the fourth-order symmetric tensor (3.8) under the orthogonal group. 
In a similar way we can construct an integrity basis for a tensor of order greater than four. For instance, we consider a tensor $\underset{\sim}{A}$ of order six as a linear operator, i.e.

$$
\mathrm{U}_{i j k}=A_{i j k l m n} T_{1 m n} .
$$

Then we can also use the representation (3.3b) where $\alpha, \beta=1,2, \ldots, 27$, and find by analogy of (3.4):

$$
\left(A_{i j k l m n}-\lambda A_{i j k l m n}^{(0)}\right) T_{1 m n}=o_{i j k} .
$$

In (3.14) the zero power tensor $A_{i j k 1 \mathrm{~m}}^{(0)}$ of order six is given by $\delta_{i 1} \delta_{j m} \delta_{k n}$, and the principal invariants can be found by the operation (3.7) where $n=27$.

In continuum mechanics a tensor $\underset{\sim}{A}$ or order six is often used as a bilinear operator, that is, instead of (3.13) we have bilinear transformations, like

$$
\mathrm{U}_{i j}=A_{i j k 1 \mathrm{mn}} \mathrm{S}_{k 1} \mathrm{~T}_{\mathrm{mn}} \text {, }
$$

which appear in constitutive expressions [14].

Now by analogy to $(3.8 \mathrm{a}, \mathrm{b})$, the usual symmetry conditions are given by

$$
A_{i j k 1 m n} \equiv A_{\alpha \beta \gamma}=A_{B \gamma \alpha}=A_{\gamma \alpha \beta}=A_{\alpha \gamma \beta}=A_{B \alpha \gamma}=A_{\gamma \beta \alpha}
$$

where $\alpha, \beta, \gamma=1,2, \ldots, 6$. From (3.15), in connection with

$$
U_{i j}=\lambda S_{i p} T_{p j}=\lambda \delta_{i k} \delta_{j n} \delta_{1 m} S_{k 1} T_{m n}
$$

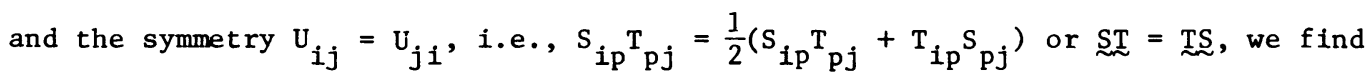
instead of (3.4b) the homogeneous system

$$
\left(A_{\alpha B}-\lambda A_{\alpha B}^{(0)}\right) V_{B}=0 ; \alpha=1,2, \ldots, 6 ; B=1,2, \ldots, 21,
$$

where $A_{\alpha B}$ is a $6 \times 21$ rectangular matrix, and $v_{B}$ with $B=1,2, \ldots, 21$ is the image of a fourth-order symmetric tensor product:

$$
\mathrm{v}_{\mathrm{B}} \equiv \mathrm{S}_{\mathrm{kl}} \mathrm{T}_{\mathrm{mm}}=\mathrm{S}_{1 \mathrm{k}} \mathrm{T}_{\mathrm{mn}}=\mathrm{S}_{\mathrm{kl}} \mathrm{T}_{\mathrm{nm}}=\mathrm{S}_{\mathrm{mn}} \mathrm{T}_{\mathrm{kl}} \text {. }
$$

Considering the symmetry relations $(3.16)$, the unit matrix $\stackrel{\sim}{(}^{(0)}$ in $(3.18)$ can be represented by

$$
\begin{aligned}
A_{i j k l m}^{(0)}= & \frac{1}{8}\left(\delta_{i 1} \delta_{j m} \delta_{k n}\right. \\
& +\delta_{i n} \delta_{j 1} \delta_{k m}+\delta_{i 1} \delta_{j n} \delta_{k m} \\
& \delta_{i m} \delta_{j 1} \delta_{k n}+\delta_{i k} \delta_{j m} \delta_{1 n}+\delta_{i k} \delta_{j n} \delta_{1 m} \\
& \left.\delta_{i m j k} \delta_{j k} \delta_{1 n}+\delta_{i n} \delta_{j k} \delta_{1 m}\right) .
\end{aligned}
$$


Because $6<21$ a nontrivial solution of the homogeneous equation (3.18) exists.

4. CONSTRUCTION OF SIMULTANEOUS INVARIANTS.

In the case of several tensor variables, the term simultaneous or joint invariant is used [6]. This term is used not only for scalar-valued isotropic functions of several second-order tensor variables, but also for scalar-valued functions of any set of tensors of any order, e. $g$. of the argument tensors $\sigma_{i j}$, $A_{i j}, A_{i j k l}, A_{i j k 1 m n}$ in (3.1).

Such invariants are, for instance,

$$
\begin{aligned}
& \sigma_{i j} A_{j i}, \sigma_{i j} A_{j k} A_{k i}, \sigma_{i k} \sigma_{k j} A_{j i}, \sigma_{i k} \sigma_{k j} A_{j 1} A_{1 i}, \\
& \sigma_{i j} A_{i j k 1} \sigma_{k 1}, \ldots, \sigma_{i j} A_{i j p q} \ldots A_{v w k 1} \sigma_{k 1} \text {, }
\end{aligned}
$$

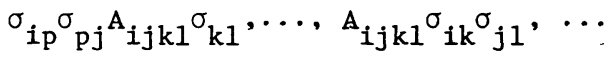

$$
\begin{aligned}
& A_{i j k 1 m n}{ }^{\sigma}{ }_{i j} \sigma_{k 1}{ }_{m n}, A_{i j p q r s} A_{k 1 t u p q} A_{m n r s t u}{ }^{\sigma}{ }_{i j}{ }^{\sigma_{k 1}{ }^{\sigma}}{ }_{m} \text {, } \\
& A_{i j k l m n} \sigma_{i l} \sigma_{j m} \sigma_{k n}, \cdots \cdot
\end{aligned}
$$

and they have great importance in the theory of anisotropic materials.

To construct a set of sumultaneous invariants of the stress tensor $\sigma_{i j}$ and the fourth-order constitutive tensor $A_{i j k 1}$ we start from the following theorem:

A scalar-valued function $f(x, T)$ of one $n$-dimensional vector $\underset{\sim}{\sim}$ and one symmetric second-order tensor $\mathrm{T}$ is an orthogonal invariant, i.e. invariant under the orthogonal group if and only if it can be expressed as a function of the $2 \mathrm{n}$ special invariants

$$
\mathrm{J}_{1}(\mathrm{~T}), \ldots, \mathrm{J}_{\mathrm{n}}(\mathrm{T}), \mathrm{v}^{2}, \mathrm{v} \cdot \mathrm{Ty}, \ldots, \mathrm{v} \cdot \stackrel{\mathrm{T}}{\mathrm{T}}^{\mathrm{T}-1} \mathrm{v}
$$

This theorem is valid for arbitrary dimension $\mathrm{n}$ [6]. Assuming the symmetry conditions (3.8) and using the HAMILTON-CAYLEY theorem, it means that ${\underset{\mathrm{T}}{\mathrm{n}}}^{\mathrm{n}}$ and all higher powers ${\underset{\sim}{\mathrm{T}}}^{\mathrm{n}+\mathrm{h}}$ can be expressed in terms of $\underset{\sim}{\delta}, \underset{\sim}{\mathrm{T}},{\underset{\sim}{\mathrm{T}}}^{2}, \ldots, \mathrm{T}^{\mathrm{n}-1}$, we find in generalizing of $(4.4)$ the set of 15 simultaneous invariants

$$
\begin{aligned}
& \Pi_{2}^{[\nu]} \equiv \sigma_{i j} A_{i j k 1}^{(\nu)} \sigma_{k 1}, \quad \Pi_{3}^{[\nu]} \equiv \sigma_{i j} A_{i j k 1}^{(\nu)} \sigma_{k 1}^{(2)}, \\
& \Pi_{4}^{[\nu]} \equiv \sigma_{i j}^{(2)} A_{i j k 1}^{(\nu)} \sigma_{k 1}^{(2)}, \quad \nu=1,2, \ldots, 5,
\end{aligned}
$$

where $v$ in a square bracket denotes a label to indicate $v$ several invariants, 
while $\nu$ in a round bracket is an exponent.

Some of these simultaneous invariants can be constructed in the following way. Consider the second-order tensor $D_{p q} \equiv \sigma_{i j} A_{i j p q}$, then, for instance, the basic invariant $\mathrm{D}_{\mathrm{pq}} \mathrm{D}_{\mathrm{qp}}$ can be expressed by the simultaneous invariant $\sigma_{i j} \mathrm{~A}_{i j \mathrm{k} 1}^{(2)} \sigma_{\mathrm{k} l}$, which is contained in the system (4.5). Furthermore, the cubic basic invariant $\mathrm{D}_{\mathrm{pq}} \mathrm{D}_{\mathrm{qr}}{ }^{\mathrm{D}} \mathrm{rp}$ can be expressed by the simultaneous invariant $A_{i j k 1 \mathrm{mn}} \sigma_{i j} \sigma_{k 1} \sigma_{\mathrm{mm}}$, which is contained in the set (4.3). As another example we can consider the second-order tensor $E_{p q} \equiv \sigma_{i j}^{(2)} A_{i j p q}$ and find, for instance, the invariant $E_{p q} E_{q p}=\sigma_{i j}^{(2)} A_{i j k 1}^{(2)} \sigma_{k 1}^{(2)}$, which is contained in (4.5), too. Finally, we can also form the simultaneous invariants

$$
\begin{aligned}
& \mathrm{D}_{p q} \sigma_{p q}=\sigma_{i j} A_{i j p q} \sigma_{p q}, \quad D_{p q} \sigma_{p q}^{(2)}=\sigma_{i j} A_{i j p q} \sigma_{p q}^{(2)} \text {, } \\
& \mathrm{E}_{\mathrm{pq}} \mathrm{\sigma}_{\mathrm{pq}}=\sigma_{i j}^{(2)} \mathrm{A}_{i j p q}{ }^{\sigma} \mathrm{pq}, \quad \mathrm{E}_{\mathrm{pq}} \sigma_{\mathrm{pq}}^{(2)}=\sigma_{i j}^{(2)} \mathrm{A}_{i j p q} \sigma_{\mathrm{pq}}^{(2)} \text {, }
\end{aligned}
$$

and obtain simultaneous invariants of the system (4.5).

The examples mentioned above and the complete system (4.5) can be expressed by the simultaneous invariants

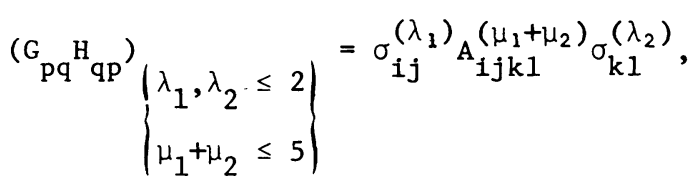

where

$$
\left(\mathrm{G}_{\mathrm{pq}}\right)_{\lambda_{1}, \mu_{1}} \equiv \sigma_{i j}^{\left(\lambda_{1}\right)} \mathrm{A}_{\mathrm{ijpq}}^{\left(\mu_{1}\right)}, \quad\left(\mathrm{H}_{\mathrm{qp}}\right){ }_{\lambda_{2}, \mu_{2}} \equiv \mathrm{A}_{\mathrm{pqk} 1}^{\left(\mu_{2}\right)} \sigma_{\mathrm{kl}}^{\left(\lambda_{2}\right)}
$$

are second-order tensors.

The isotropic special case, for instance, can be expressed by the isotropic constitutive tensor

$$
A_{i j k 1}^{(\nu)}=a_{v} \delta_{i j k 1} \delta_{k}+b_{\nu}\left(\delta_{i k} \delta_{j 1}+\delta_{i 1} \delta_{j k}\right)
$$

of power $\nu=1,2, \ldots$. Then the simultaneous invariants $\Pi_{2}^{[\nu]}$ from (4.5) are equal to the principal invariant $\mathrm{J}_{2}(\underset{\sim}{)})$ for $a_{V}=-\frac{1}{2}, b_{V}=\frac{1}{4}$ and equal to the basic invariant $\mathrm{S}_{2}(\underset{\sim}{)})$ for $a_{\nu}=0, \mathrm{~b}_{\nu}=\frac{1}{2}$. Similarly, the invariants $\Pi_{3}^{[\nu]}$ from $(4.5)$ are equal to $\mathrm{J}_{3}(\sigma)-\mathrm{J}_{1}^{3}(\sigma) / 6$ for $\mathrm{a}_{v}=-\frac{1}{2}, \mathrm{~b}_{v}=\frac{1}{6}$ and equal to the cubic basic 
invariant $s_{3}(\sigma)$ if $a_{\nu}=0, b_{\nu}=\frac{1}{2}$. Furthermore, the invariants $\Pi_{1}^{[\nu]} \equiv \delta_{i j} A_{1 j k 1}^{(\nu)} \sigma_{k 1}$ are equal to $\mathrm{J}_{1}(\sigma) \equiv \mathrm{s}_{1}(\sigma)$ if $3 \mathrm{a}_{v}+2 \mathrm{~b}_{v}=1$.

\section{REFERENCES}

1. GRACE, J.H. and YOUNG, A., The Algebra of Invariants, Cambridge Univ. Press, London and New York, 1903.

2. GUREVICH, G.B., Foundations of the Theory of Algebraic Invariants, P. Noordhoff, Groningen, 1964.

3. WEITZENBOCK, R., Invariantentheorie, P. Noordhoff, Groningen, 1923.

4. WEYL, H., The Classical Groups, Their Invariants and Representation, Princeton Univ. Press, Princeton and New Jersey, 1946.

5. SPENCER, A.J.M., Theory of Invariants, in: Continuum Physics. Edited by A.C. Eringen, Vol. 1 Mathematics, Academic Press, New York and London, 1971.

6. TRUESDELL, C. and NOLL, W., The non-1inear Field Theories of Mechanics, in: Handbuch der Physik. (Edited by S. Flugge), Vol. III/3, Springer-Verlag, Berlin/Heidelberg/New York, 1965.

7. BETTEN, J., Ein Beitrag zur Invariantentheorie in der Plastomechanik anisotroper Werkstoffe, Z. Angew. Math. Mech. 56 (1976), 557-559.

8. SMITH, G.F., On the Yield Condition for Anisotropic Materials, Quart. App1. Math. 20 (1962), 241-247.

9. SPENCER, A.J.M. and RIVLIN, R.S., The Theory of Matrix Polynomials and its Application to the Mechanics of Isotropic Continua, Arch. Rational Mech. Anal. 2 (1958/59), 309-336.

10. WINEMAN, A.S. and PIPKIN, A.C., Material Symmetry Restrictions on Constitutive Equations, Arch. Rational Mech. Anal. 17 (1964), 184-214.

11. SMITH, G.F., SMITH, M.M., and RIVLIN, R.S., Integrity Bases for a Symmetric Tensor and a Vector - The Crystal Classes, Arch. Rational Mech. Anal. 12 (1963), 93-133.

12. PIPKIN, A.C. and RIVLIN, R.S., The Formulation of constitutive equations in continuum physics. Arch. Rational Mech. Anal. 4 (1959), 129-144.

13. PIPKIN, A.C. and WINEMAN, A.S., Material Symmetry Restrictions on Non-Polynomial Constitutive Equations, Arch. Rational Mech. Anal. 12 (1963), 420-426.

14. BETTEN, J., Plastische Stoffleichungen inkompressibler anisotroper Werkstoffe, Z. Angew. Math. Mech. 57 (1977), 671-673. 


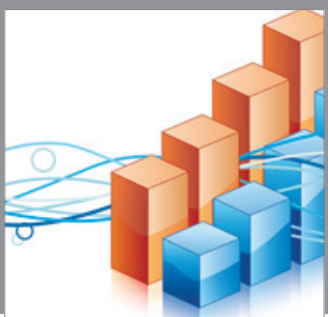

Advances in

Operations Research

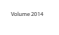

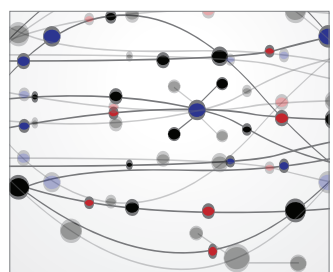

\section{The Scientific} World Journal
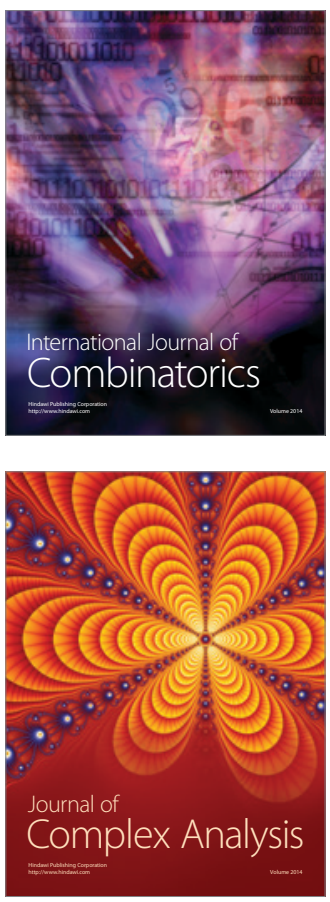

International Journal of

Mathematics and

Mathematical

Sciences
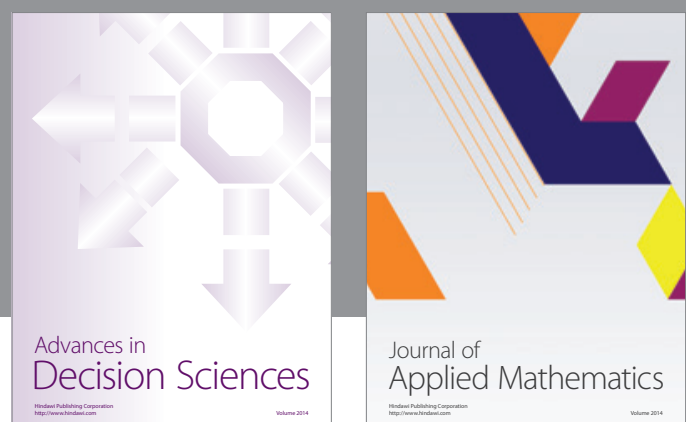

Journal of

Applied Mathematics
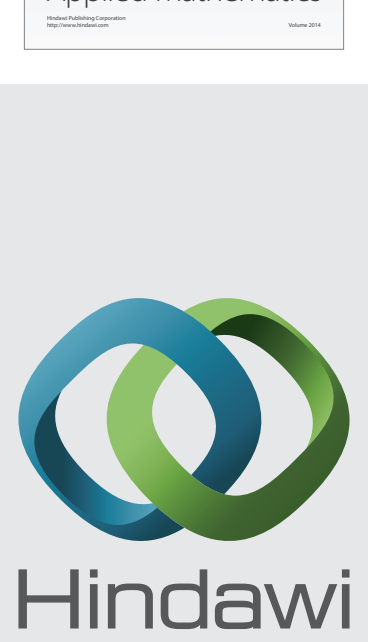

Submit your manuscripts at http://www.hindawi.com
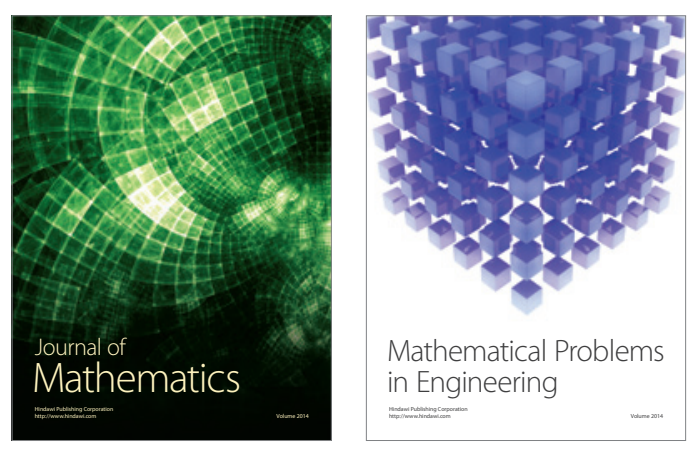

Mathematical Problems in Engineering
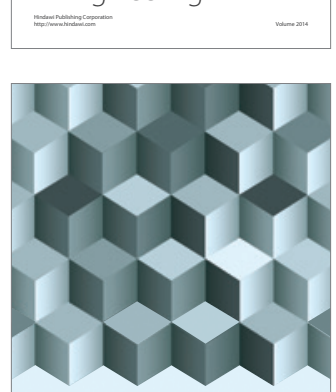

Journal of

Function Spaces
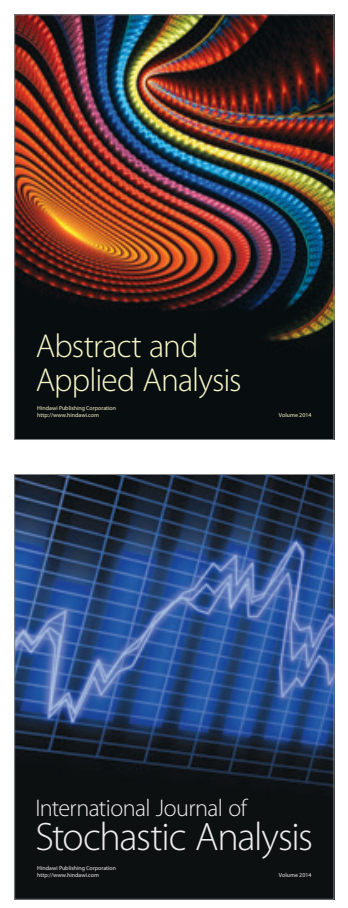

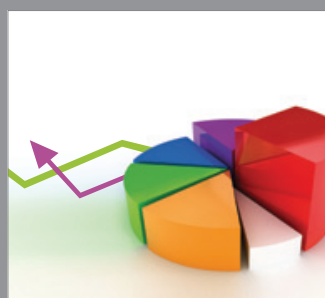

ournal of

Probability and Statistics

Promensencen
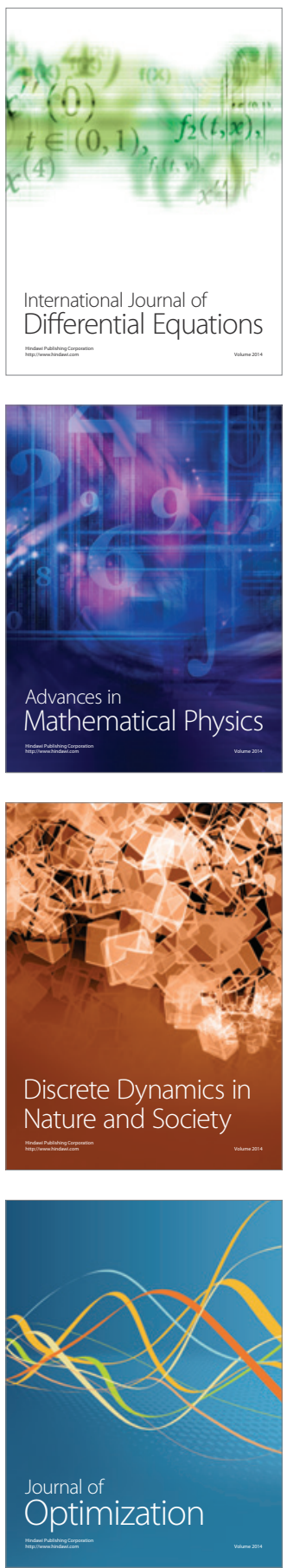